\title{
Craniofacial Fibrous Dysplasia of the Mandible: A Case Report and Literature Review
}

\author{
Nicolás Yáñez ${ }^{1}$, Maximiliano Bravo² and Francisco Javier Pinedo ${ }^{3,4,5^{*}}$ \\ ${ }^{1}$ Doctor in Dental Surgery, Pontificia Universidad Católica de Chile, Santiago, Chile \\ ${ }^{2}$ Oral and Maxillofacial Surgery Resident, Universidad de los Andes, Santiago, Chile
}

${ }^{3}$ Associate Instructor, Oral and Maxillofacial Surgery Department, Faculty of Medicine, Dentistry School, Universidad Católica de Chile, Santiago, Chile

${ }^{4}$ Maxillofacial Surgery Department, Hospital Santiago Oriente - Dr. Luis Tisné Brousse, Santiago, Chile

${ }^{5}$ Dentistry Department, Oral and Maxillofacial Surgeon, Clínica Santa María, Santiago, Chile

*Corresponding author: Francisco Javier Pinedo, Associate Instructor, Oral and Maxillofacial Surgery Department, Faculty of Medicine, Dentistry School, Universidad Católica de Chile, Santiago, Chile; Maxillofacial Surgery Department, Hospital Santiago Oriente - Dr. Luis Tisné Brousse, Santiago, Chile; Dentistry Department, Oral and Maxillofacial Surgeon, Clínica Santa María, Santiago, Chile, Tel: +56-9-9405-4577

\begin{abstract}
Objective: Report a case of craniofacial fibrous dysplasia of the mandible with a literature review about the management of this disease.

Clinical case: A 47-years-old female patient was referred to our unit due to an incidental radiographic finding of a mixed radiolucent-radiopaque lesion in the right mandible ramus. Complementary blood tests, an updated panoramic radiography and a cone beam computed tomography were performed. The incisional biopsy's histopathological report described a craniofacial monostotic fibrous dysplasia of the mandible.

Discussion: The fibrous dysplasia is a low prevalence fibroosseous lesion that can affect one or more bones, causing different degree of alterations. Treatment must be assessed according to several factors like age, location, size, aggressiveness, among others. Conservative management with long-term follow-up is the treatment of choice in certain cases, avoiding surgical morbidity.

Conclusion: The craniofacial fibrous dysplasia is a rare disease of which diagnosis is difficult due to multiple differential diagnosis. The conservative or surgical management must be assessed depending on factors such as aggressiveness, synthomathology and presence of aesthetics and/or functional alterations.
\end{abstract}

\section{Keywords}

Monostotic fibrous dysplasia, Craniofacial fibrous dysplasia, Bone disease

\section{Introduction}

Fibro-osseous lesions of the jaws are a group of benign disorders that can affect bone. Within this group is Fibrous Dysplasia (FD), whose name was mentioned for the first time by Lichtenstein in 1938 [1] and then by Lichtenstein and Jaffe in 1942 [2].

In this condition normal bone tissue is replaced by fibrous tissue and immature bone characterised by abnormal proliferation of fibrous tissue between normal and immature bone, caused by poorly differentiated osteoblasts. Its etiology remains unknown [3]. It represents $3 \%$ of all bone tumors and between $5-7 \%$ of all benign bone tumors $[4,5]$.

In decreasing order, its most frequent locations are the maxilla, mandible and frontal bone $[6,7]$. It commonly involves adjacent structures such as dental arch, maxillary sinus, airway, orbit, and skull base $[8,9]$.

Regarding the alterations associated with its presence in the mandibular region, several signs and symptoms have been described such as neurosensitive disturbances due to compression of the inferior alveolar nerve canal [10-13], malocclusion, facial deformity, among others [14].

Citation: Yáñez N, Bravo M, Pinedo FJ (2021) Craniofacial Fibrous Dysplasia of the Mandible: A Case Report and Literature Review. Res Rep Oral Maxillofac Surg 5:053. doi.org/10.23937/26433907/1710053

Accepted: August 03, 2020; Published: August 05, 2021

Copyright: (C) 2021 Yáñez N, et al. This is an open-access article distributed under the terms of the Creative Commons Attribution License, which permits unrestricted use, distribution, and reproduction in any medium, provided the original author and source are credited. 

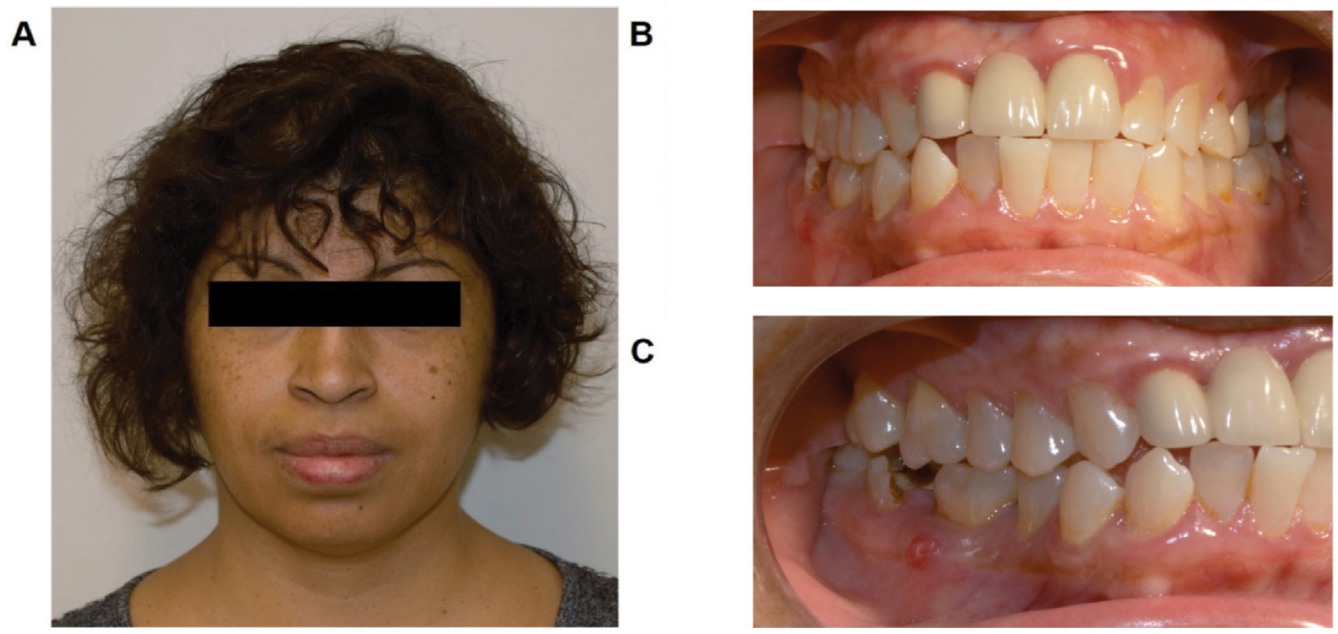

Figure 1: A) Clinical photos upon admission of the patients; B) Front view of the oral cavity; C) Lateral view of the oral cavity. Note the presence of a fistula in relation to tooth 46 and caries in tooth 47 .

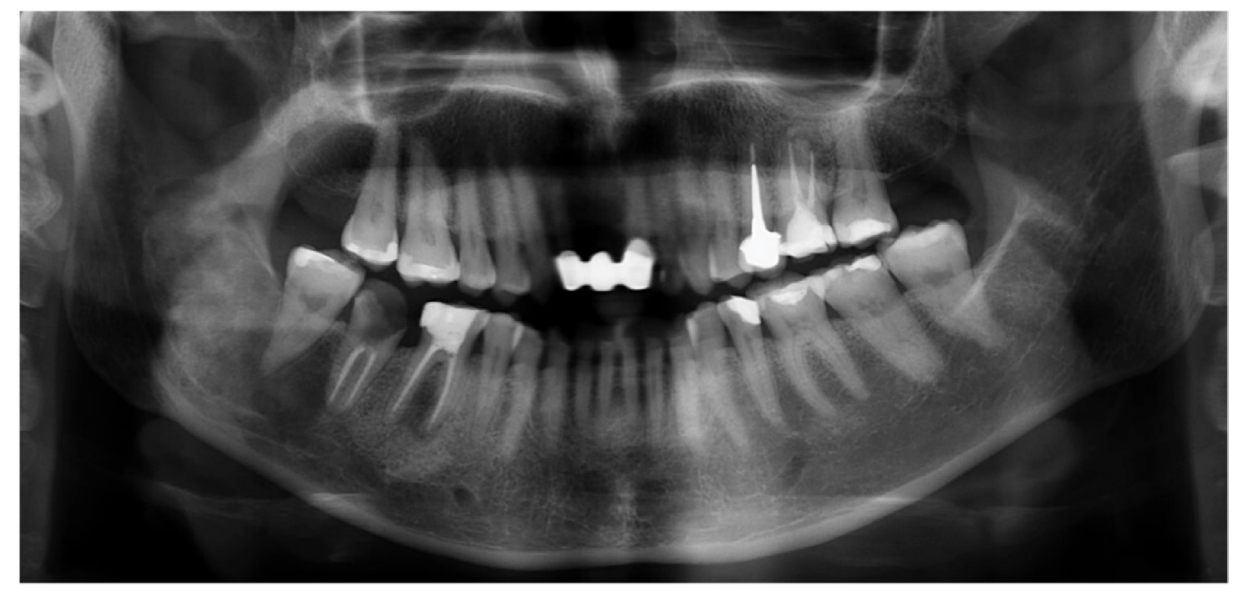

Figure 2: Orthopantomography. A lesion is observed in the body and right mandibular branch in a ground glass pattern.

The aim of this study is to report a case of monostotic FD in the mandible and review the current literature about the management of this lesion.

\section{Case Description}

A 47-year-old female referred for dental care to the Dentistry Department of Santa María Clinic reported an approximately 7 months of evolution pain in the right mandibular body. In a previously performed orthopantomography there was an large lesion. She does not declare any morbid, allergic or surgical history. On physical examination, no major findings or alterations are described (Figure 1A). On oral examination, the midline is displaced to the left (Figure 1B). Likewise, a chronic infection is evidenced in relation to teeth 46 and 47 associated with failed previous treatments (Figure 1C). There is no expansion of the mandibular cortical tables. Complete blood count, calcium, phosphorus, serum creatinine, alkaline phosphatase, thyroid stimulating hormone (TSH), free thyroxine (T4) and Parathormone (PTH) levels are requested, without observing altered values.
In the orthopantomography (Figure 2), a heterogeneous, radiolucent/mixed-aspect lesion is evidenced that involves part of the body and mandibular branch on the right side.

A Cone Beam Computed Tomography (CBCT) was taken, which revealed an extensive and irregular hypodense area, with clear limits, which involves the middle and lower third of the ramus and mandibular body from the right side to the distal side of tooth 46 , compromising the entire alveolar process alveolar from the crest to the mandibular canal. It displaces the mandibular canal towards the basilar edge, generating a solution of continuity in the canal roof. It expands buccal and lingual bone tables, without drilling or thinning the bone cortex, does not displace teeth or generate rhizalysis (Figure 3).

Our diagnostic hypotheses were calcifying epithelial odontogenic tumor, ossifying fibroma, and fibrous dysplasia. Through an incisional biopsy (Figure 4), a histopathological study was performed where the definitive diagnosis of Monostotic Fibrous Dysplasia of the Mandibular bone was established. 


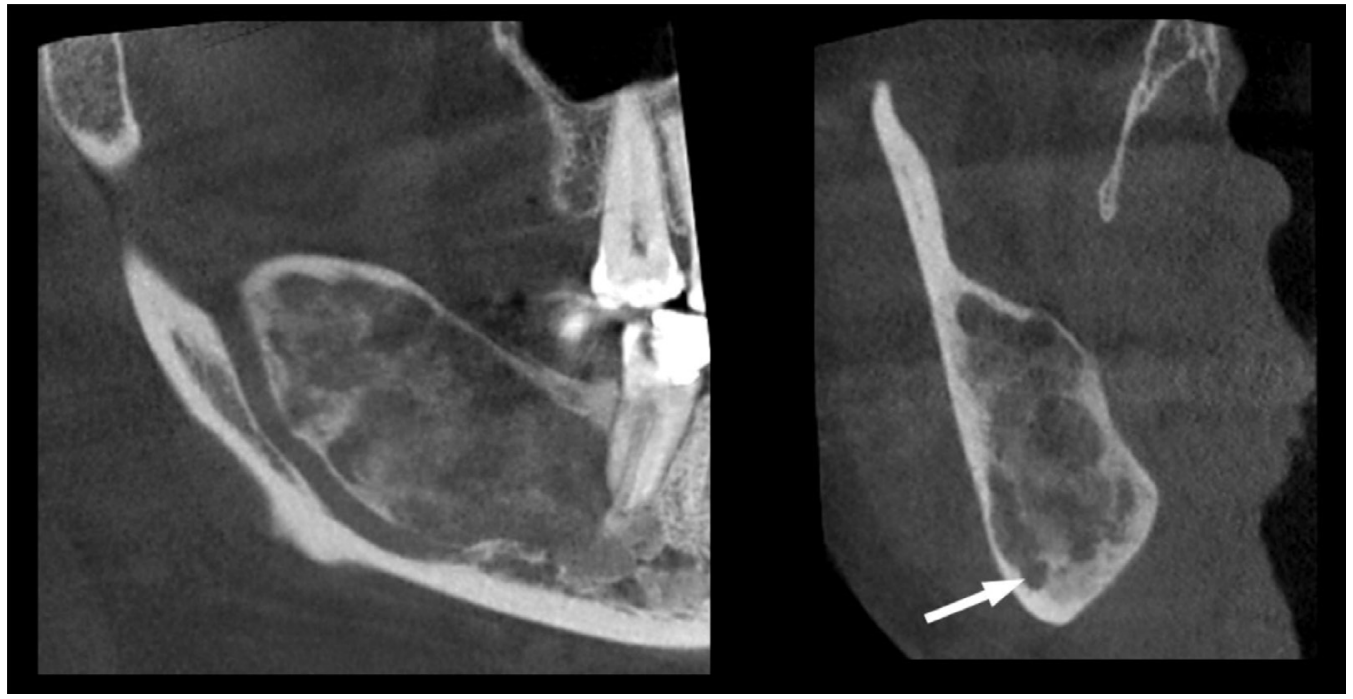

Figure 3: Sagittal and coronal sections of CBCT. Arrow shows caudal displacement of the mandibular canal.

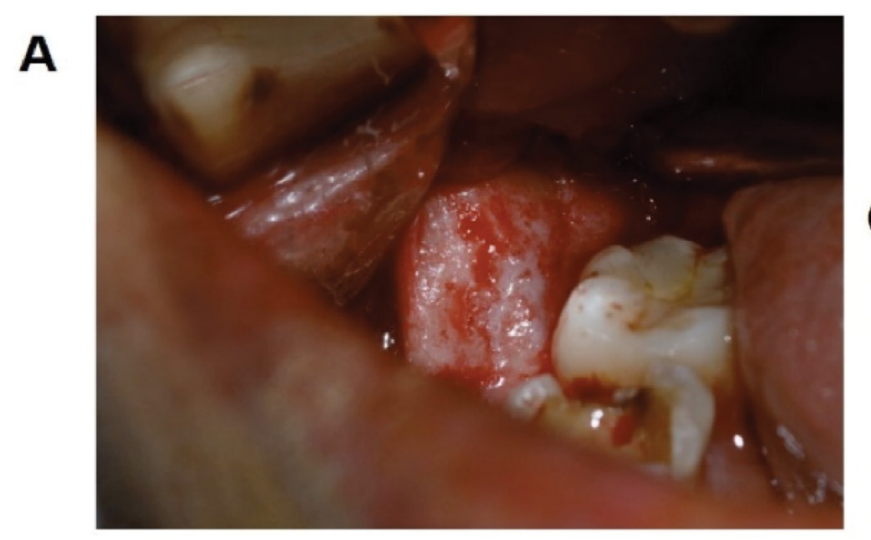

B
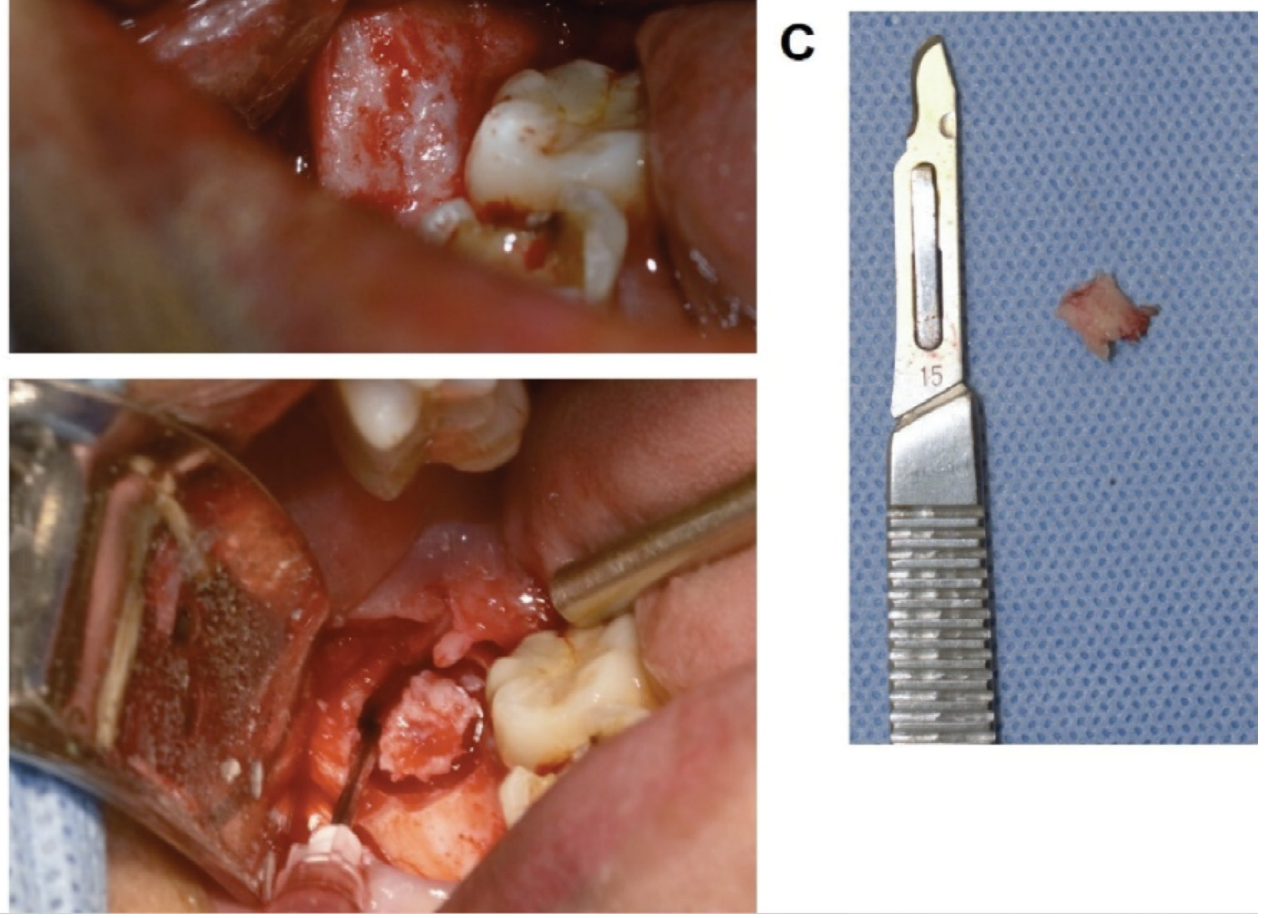

Figure 4: Intraoperative images of the incisional biopsy. A) Exposure; B) Sample margins; C) Tissue extracted for histopathological study.

\section{Dicussion}

The latest WHO head and neck tumor classification, published in 2017, located FD in the group of "osteochondromatous and fibroosseus lesions [15].

It can occur at any age, although it is more frequent in children and adults between the first and second decade [5], without predilection for sex $[5,11]$. There are also no clear conclusions regarding its progress and arrest, although most studies imply a decrease in the rate of progression or remission after puberty $[5,16]$.

Regarding the location, its incidence in the maxilla is almost double that in the mandible. In the maxilla, it usually presents unilaterally in its posterior region $[7,17]$. In this case, the lesion affected the mandibular branch and body on the right side.

It usually has a slow and progressive growth with associated complications of pain, facial deformity, fractures, functional impotence, dental and neurological 
disturbances $[18,19]$. Reports of FD located in the mandibular region have been observed with prevalences between 25 and 50\% [7,20].

Genetically it is expressed in two ways, monostotic (56-70\%) or polyostotic (30-47\%) [5,7,21-23]. The most frequent location for FD in descending order is the maxilla, mandibule and frontal bones $[7,18]$. They commonly involve adjacent structures, such as the dental arch, maxillary sinus, airway, orbit, and skull base.

Malignancy is rare, being approximately $0.5 \%$ in the monostotic variable and 4\% for SMA. This situation has been seen with radiation therapy and growth hormone (GH) alterations. [4,5,24].

Its differential diagnoses include Paget's disease, type I neurofibromatosis, osteofibrous dysplasia, ossifying fibroma, cherubism, osteosarcoma among others [22]. These lesions present a wide clinical spectrum of expression, from being practically asymptomatic to a disabling alteration for the senses such as hearing and vision.

Considering the high rate of casual radiographic findings of the lesion [25], we did not find reports describing the appearance of monostotic or polyostotic craniofacial lesions in conjunction with lesions in other parts of the body.

The diagnosis of FD represents a challenge in clinical practice, it requires clinical, radiological, histopathological correlation and surgical findings [26]. Although the clinical characteristics can provide a diagnostic approach for this type of lesion, they are not specific to each other, so histopathology is key for a definitive diagnosis and proper management of the lesions. In our case, prior to performing the biopsy, the first differential diagnoses pointed to an odontogenic tumor or cyst due to the higher prevalence of these lesions over the fibro-osseous ones, in addition to its unusual location.

Although histopathology is important to establishing a definitive diagnosis, sometimes it is inconclusive. This is due to the great similarity between the histopathological characteristics with other fibro-osseous lesions such as ossifying fibroma. It is important to consider the stage of maturation of the lesion and the variations in its histological patterns for the establishment of a definitive diagnosis. The gold standard for the diagnosis of FD is the histopathological confirmation of a fibro-osseous lesion without a capsule, which must be confirmed with radiographic findings [11]. Therefore, close collaboration between the surgeon and the pathologist is essential for the establishment of a definitive diagnosis.

The treatment of FDs must be highly individualized and depends on their clinical presentation [27]. Treatment protocols include observation, medical treatment, and/or surgery. The age, affected site, size and the biological behavior of the lesion are influencing factors in the selection of therapeutic management [28]. In relation to the biological behavior, lesions of the facial skeleton can be described as quiescent, nonaggressive or aggressive.

The first line of treatment is surgical, which can be conservative or invasive, through resection and subsequent reconstruction $[5,21,29,30]$. It has also been determined that observation and constant monitoring is feasible in incidentally found lesions, without signs or symptoms associated. Recurrence of FD is common, reaching up to $50 \%$ in children until the end of growth, and is the most common cause for reoperation $[6,21]$.

On the other hand, it has been shown that therapy with intravenous bisphosphonates (Zoledronic acid or Pamidronate) and Denosumab (RANKL monoclonal antibody) have been effective in relieving pain and normalizing bone turnover. There are some studies that report the complete or partial disappearance of lesions after prolonged therapy with bisphosphonates [31,32], however, its use is recommended in the symptomatic polyostotic variable [21,31,33].

In the presence of deformity, functional impotence, risk of pathological fracture or symptoms, conservative surgical management is recommended. In more severe cases, with a greater compromise of adjacent structures, remodeling, shaving or bone grafting is also a viable alternative $[5,6]$. Treatment goals seek to correct or prevent functional problems caused by injuries and achieve aesthetic improvement. In our case, the patient did not manifest pain, deformity, functional or neurological alterations.

In the case of quiescent fibrous dysplasia in which facial deformation or relative risk of pathological fracture is not investigated, observation and follow-up is an acceptable treatment modality $[5,29]$.

In the present case, the characteristics of her pathology, prognosis and treatment options were explained to the patient. It was decided to maintain clinical, photographic and radiographic controls. Considering that the malignancy of the FD varies between $0.4 \%-4 \%$, continuous and close monitoring, clinical and radiological, every 6 months is suggested to control the evolution of the pathology [5]. The patient should be informed that if signs or symptoms appear, they should promptly inform their caregiver.

The finding of mandibular FD in the area of the body and angle in a woman in her 4th decade is uncommon. However, the general dentist may be the first to detect it in routine practice with a panoramic radiograph. Therefore, the knowledge of the pathology is essential in the conformation of an appropriate diagnosis and in reducing potential complications.

\section{Conclusion}

The diagnosis of FD is challenging for the clinician and 
requires a comprehensive clinical history and physical examination. When selecting the appropriate treatment for FD, the clinical characteristics should be considered individually for the case, considering factors such as age, the presence of comorbidities, aggressiveness, the stage of development, the associated symptoms and the presence of functional or aesthetic alterationst. Close monitoring is essential in cases where the selected treatment is observation.

\section{Ethic Aspects}

The authors declare that the study was done in accordance with the code of ethics of the World Medical Association (Declaration of Helsinki) for human studies.

\section{Conflict of Interest}

No conflicts of interest are declared.

\section{Sources of Support}

There is no source of support for this study. All authors made substantial contributions to the conception of this article.

\section{References}

1. Lichtenstein L (1938) Polyostotic Fibrous Dysplasia. Arch Surg 36: 98.

2. Lichtenstein LJH (1942) Fibrous dysplasia of bone. A condition affecting one, several or many bones, the graver cases of which may present abnormal pigmentation of skin, premature sexual development, hyperthyroidism or still other extraskeletal abnormalities. Arch Pathol 33: 777-816.

3. Godse AS, Shrotriya SP, Vaid NS (2009) Fibrous dysplasia of the maxilla. J Pediatr Surg 44: 849-851.

4. Kruse A, Pieles U, Riener MO, Zunker C, Bredell MG, et al. (2009) Craniomaxillofacial fibrous dysplasia: A 10-year database 1996-2006. Br J Oral Maxillofac Surg 47: 302305.

5. Dicaprio MR, Enneking WF (2005) Fibrous Dysplasia Pathophysiology, Evaluation, and Treatment. J Bone Jt Surg 87: 1848-1864.

6. Boyce AM, Florenzano P, Castro DF, Collins MT (2015) Fibrous Dysplasia/McCune-Albright Syndrome.

7. Yang L, Wu H, Lu J, Teng L (2017) Prevalence of Different Forms and Involved Bones of Craniofacial Fibrous Dysplasia. J Craniofac Surg 28: 21-25.

8. Hartley I, Zhadina M, Collins MT, Boyce AM (2019) Fibrous Dysplasia of Bone and McCune-Albright Syndrome: A Bench to Bedside Review. Calcif Tissue Int 104: 517-529.

9. El-Naggar A, Chan J, Takata T, Grandis J, Slootweg P (2017) The Fourth Edition of the Head and Neck World Health Organization Blue Book: Editors' Perspectives. Hum Pathol 66: 10-12.

10. Wei WB, Chen MJ, Yang C, Zhang W, Wang Y (2015) Decompression of the inferior alveolar nerve to treat the pain of the mandible caused by fibrous dysplasia-case report. Int J Clin Exp Med 8: 19535-19539.

11. Chen YR, Chang C, Tan Y (2006) Craniofacial Fibrous Dysplaisa: An Update. Chang Gund Med J 29: 543-549.

12. Brown EW, Megerian CA, Mckenna MJ, Weber A (1995)
Fibrous Dysplasia of the Temporal Bone: Imaging Findings. 164: 679-682.

13. Cai M, Ma L, Xu G, Gruen P, Li J, et al. (2012) Clinical and radiological observation in a surgical series of 36 cases of fibrous dysplasia of the skull. Clin Neurol Neurosurg 114: 254-259.

14. Akintoye SO, Lee JS, Feimster T, Booher S, Brahim J, et al. (2003) Dental characteristics of fibrous dysplasia and McCune-Albright syndrome. Oral Surg Oral Med Oral Pathol Oral Radiol Endod 96: 275-282.

15. Florez H, Peris P, Guañabens N (2016) Displasia fibrosa. Revisión clínica y abordaje terapéutico. Med Clin (Barc) 147: 547-553.

16. Frodel JL, Funk G, Boyle J, Richardson M (2000) Management of Aggressive Midface and Orbital Fibrous Dysplasia. Arch Facial Plast Ssurgery 2: 187-195.

17. MacDonald-Jannkowski D (1999) Fibrous Dysplasia in the Jaws of a Hong-Kong Population: Radiographic Presentation and Systematic Review. Dentomaxillofacial Radiol 28: 195-202.

18. Boyce A, Burke AB, Cutler Peck C, DuFresne CR, Lee JS, et al. (2015) Indications and Long-Term Outcomes of Surgical Treatment for Craniofacial Fibrous Dysplasia. J Oral Maxillofac Surg 73: 49-50.

19. Sweeney K, Kaban LB (2019) Natural History of Fibrous Dysplasia and McCune-Albright Syndrome. J Oral Maxillofac Surg 77: e15-e16.

20. Nager G, Kenedy D, Kopsteinn E (1982) Fibrous Dysplasia: A Review of the Disease and Its Manifestations in the Temporal Bone. Ann Otol Rhinol Laryngol 92: 1-52.

21. Oualha L, Omezzine M, Douki N, Khochtalli H (2017) Fibrous dysplasia in the maxillomandibular region: retrospective study. Int J Oral Maxillofac Surg 46: 310.

22. Valentini V, Cassoni A, Marianetti TM, Terenzi V, Fadda MT, et al. (2009) Craniomaxillofacial Fibrous Dysplasia: Conservative Treatment or Radical Surgery? A Retrospective Study on 68 Patients. Plast Reconstr Surg 123: $653-660$.

23. Wu H, Yang L, Li S, Jin X, Xu J, et al. (2014) Clinical characteristics of craniomaxillofacial fibrous dysplasia. $\mathrm{J}$ Cranio-Maxillofacial Surg 42: 1450-1455.

24. Chen M, Yang C, Fang B, Zhang S, Xu K (2010) Treatment of Hemimandibular Fibrous Dysplasia with Radical Excision and Immediate Reconstruction with Free Double Costochondral Graft. J Oral Maxillofac Surg 68: 2000-2004.

25. Delilbasi C, Deniz E, Ekici ID (2014) Monostotic Fibrous Dysplasia of the Mandible. Oral Heal Dent Manag 13: 326329.

26. Biermann JS (2002) Common Benign Lesions of Bone in Children and Adolescents. J Pediatr Orthop 22: 268-273.

27. Brannon RB, Fowler CB (2001) Benign fibro-osseous lesions: A review of current concepts. Adv Anat Pathol 8: 126-143.

28. Panda NK, Parida PK, Sharma R, Jain A, Bapuraj JR (2007) A clinicoradiologic analysis of symptomatic craniofacial fibro-osseous lesions. Otolaryngol-Head Neck Surg 136: 928-933.

29. Mäkitie AA, Törnwall J, Mäkitie O (2008) Bisphosphonate treatment in craniofacial fibrous dysplasia - A case report and review of the literature. Clin Rheumatol 27: 809-812.

30. Chapurlat RD (2007) Medical Therapy in Adults with Fibrous Dysplasia of Bone. J Bone Miner Res 21: 114-119. 
31. Lee JS, Fitzgibbon EJ, Chen YR, Kim HJ, Lustig LR, et al. (2012) Clinical guidelines for the management of craniofacial fibrous dysplasia. Orphanet Journal of Rare Diseases 7: S2.

32. Marx RE, Sawatari Y, Fortin M, Broumand V (2005) Bisphosphonate-induced exposed bone (osteonecrosis/ osteopetrosis) of the jaws: Risk factors, recognition, prevention, and treatment. J Oral Maxillofac Surg 63: 15671575.

33. Regezi JA, Sciubba JJ, Jordan RCK (2003) Oral Pathology: Clinical Pathologic Correlations. $\left(6^{\text {th }}\right.$ edn), Springer Nature, 212. 